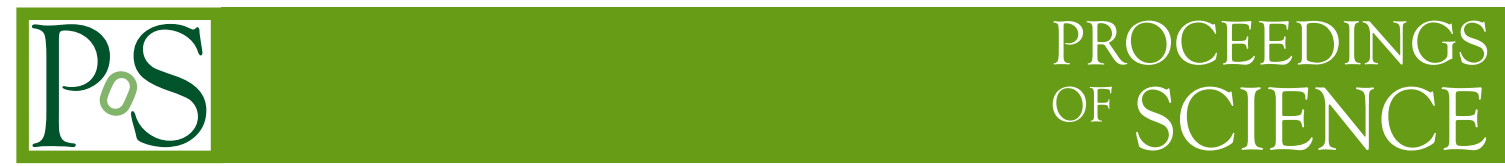

\title{
Search for New Physics in All-Hadronic Final States at CMS
}

\section{Kai Yi on behalf of the CMS Collaboration*}

Physics and Astronomy Department, University of Iowa, Iowa, USA

E-mail: yikefnal.gov

Searches for new physics in hadronic final states are performed using data up to $1 \mathrm{fb}^{-1}$ collected by the CMS experiment at the LHC at a collsion energy of $\sqrt{s}=7 \mathrm{TeV}$. No new physics is found and upper limits are set for various models: ADD, unparticle, string resonance, excited quarks, axigluons, Randall-Sundrum gravitons, RPV SUSY gluino, as well as black holes.

The 2011 Europhysics Conference on High Energy Physics-HEP 2011,

July 21-27, 2011

Grenoble, Rhône-Alpes France

\footnotetext{
* Speaker.
} 
Table 1: . Observed and expected $95 \% \mathrm{CL}$ lower limits on the ADD model parameter $M_{D}\left(\right.$ in $\left.\mathrm{TeV} / \mathrm{c}^{2}\right)$ as functions of $\delta$, with and without NLO K factors applied.

\begin{tabular}{r|r|rr|rr}
\hline$\delta$ & K factor & LO Exp. & LO Obs. & NLO Exp. & NLO Obs. \\
\hline 2 & 1.5 & 2.17 & 2.29 & 2.41 & 2.56 \\
3 & 1.5 & 1.82 & 1.92 & 1.99 & 2.07 \\
4 & 1.4 & 1.67 & 1.74 & 1.78 & 1.86 \\
5 & 1.4 & 1.59 & 1.65 & 1.68 & 1.74 \\
6 & 1.4 & 1.54 & 1.59 & 1.62 & 1.68 \\
\hline
\end{tabular}

\section{Jets and Missing ET Reconstruction at CMS}

Jets and missing $E_{T}$ (MET) are reconstructed using a particle flow technique [1] at CMS [2]. The CMS particle-flow algorithm uses calorimeter information and combines it with reconstructed tracks to identify individual particles such as photons, leptons, and both neutral and charged hadrons within the jets. The particle-flow objects serve as input for jet reconstruction, performed using the anti- $k_{T}$ algorithm [3] with a cone radius $R=\sqrt{(\Delta \eta)^{2}+(\Delta \phi)^{2}}$ of 0.5 or 0.7 . Jet energy scale corrections are derived from Monte Carlo (MC) simulation [4] as well as data. The missing transverse energy vector is computed as the negative vector sum of the transverse momenta of all particles reconstructed in the event, and has a magnitude denoted by MET.

\section{Search for New Physics using a Monojet plus MET Signature}

This search is focused on the search for production of a real graviton, $G$, from the ADD (Arkani-Hamed, Dimopoulos, and Dvali) model, or unparticle, $U$, balanced by an energetic hadronic jet in the missing energy plus single jet final state [5]. The data used in this study correspond to an integrated luminosity of $36 \pm 4 \mathrm{pb}^{-1}$.

The monojet signal region is selected by requiring MET $>150 \mathrm{GeV}$ and the most energetic jet in the event to have transverse momentum $p_{T}\left(j_{1}\right)>110 \mathrm{GeV} / \mathrm{c}$. Events with more than two jets with $p_{T}$ above $30 \mathrm{GeV} / \mathrm{c}$ are discarded. The main background after this selection comes from $Z(v v)+$ jets and $W+$ jets and are estimated from $W(\mu v)+$ jets data sample. The observed data is consistent with the Standard Model (SM) prediction, and no significant excess is observed.

Exclusion limits for the ADD model are given in Table 1 and significantly improve the previous limits. For unparticles with spin $=0$, production cross sections above $54 \mathrm{pb}$ are excluded at $95 \%$ confidence level (CL) for $\mathrm{dU}=1.7$ and $\Lambda_{U}=1 \mathrm{TeV} / \mathrm{c}^{2}$. The limits for other $\mathrm{dU}$ and $\Lambda_{U}$ are comparable and are shown in Fig. 1 (left). These results have since been updated with $1 \mathrm{fb}^{-1}$ [5].

\section{Dijet Resonances Searches}

This study is focused on a search for narrow resonances in the dijet mass spectrum. We consider the following models which decay to the parton pairs listed in parentheses [6]: string resonance ( $q g, q \bar{q}$ and $g g$ ); axial vector particles called axigluons $A(q \bar{q})$; color-octet coloron $C(q \bar{q})$; excited quarks $q^{*}(q g)$ expected from composite quark model; $E_{6}$ diquark ( $\bar{q} \bar{q}$ and $q q$ ) predicted 

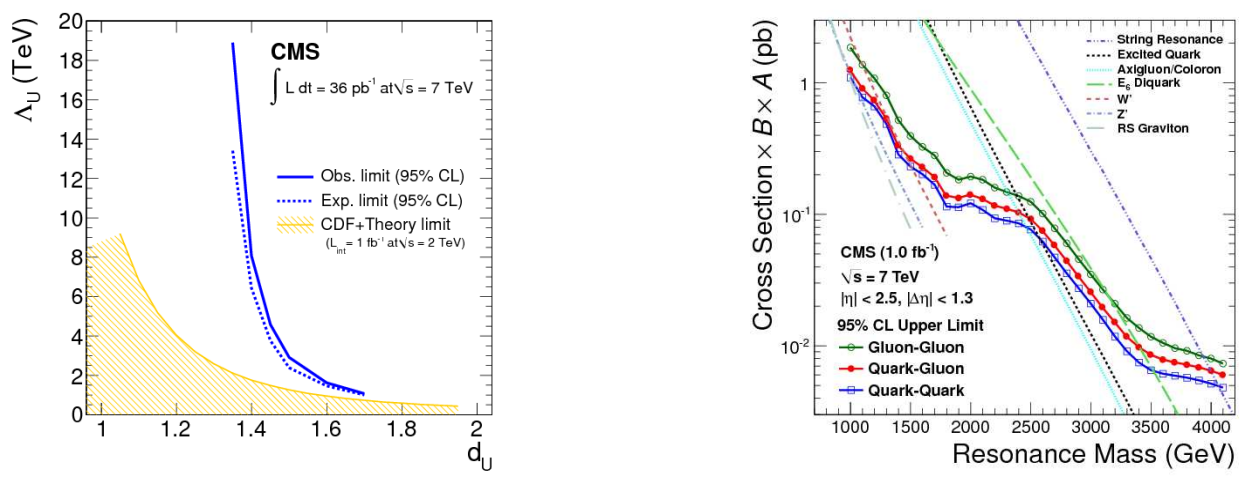

Figure 1: Left: Observed and expected limits on ADD versus the theoretical cross section and as a function of $M_{D}$. Limits are shown for $\delta=2$ and $\delta=4$. Right: The 95\% CL upper limits on $\sigma \times B \times A$ for dijet resonances of type gluon-gluon (open circles), quark-gluon (solid circles), and quark-quark (open boxes), compared to theoretical predictions for string resonances, E6 diquarks, excited quarks, axigluons, colorons, new gauge bosons $\mathrm{W}$ and Z, and Randall-Sundrum gravitons.

from grand unified theory based on the $E_{6}$ gauge group; massive gravitons $G(q \bar{q}$ and $g g$ ) from the Randall-Sundrum (RS) model of extra dimensions; new gauge bosons $W^{\prime}$ and $Z^{\prime}$ from new gauge $\operatorname{symmetries}(q \bar{q})$.

We search for narrow resonances in the smooth dijet mass spectrum using the two leading jets in the data. The background shape is modeled by a smooth parameterization. The dijet resonance shapes for $q q, q g$ and $g g$ partons are dominated by experimental resolution and are obtained from full simulation. The data used in this search correspond to an integrated luminosity of $1.1 \mathrm{fb}^{-1}$. No significant excess is observed.

The upper limits at 95\% CL set on the cross section times branching ratio times acceptance of centrally $(|\Delta \eta|<1.3$ and $|\eta|<2.5)$ produced dijet mass resonances for $(q q, q g, g g)$ are shown in Fig. 1 (right). For string resonances we exclude masses less than $4.00 \mathrm{TeV}$; this extends our previous exclusion of $0.5<M(S)<2.5 \mathrm{TeV}$. For E6 diquarks we exclude masses less than 3.52 $\mathrm{TeV}$; this extends our previous exclusions of $0.50<M(D)<0.58 \mathrm{TeV}, 0.97<M(D)<1.08 \mathrm{TeV}$, and $1.45<M(D)<1.60 \mathrm{TeV}$. For excited quarks we exclude masses less than $2.49 \mathrm{TeV}$; this extends our previous exclusion of $0.5<M\left(q^{*}\right)<1.58 \mathrm{TeV}$. For axigluons or colorons we exclude masses less than $2.47 \mathrm{TeV}$; this extends our previous exclusions of $0.50<M(A, C)<1.17 \mathrm{TeV}$ and $1.47<M(A, C)<1.52 \mathrm{TeV}$. For $\mathrm{W}$ bosons we exclude masses less than $1.51 \mathrm{TeV}$; this extends the 


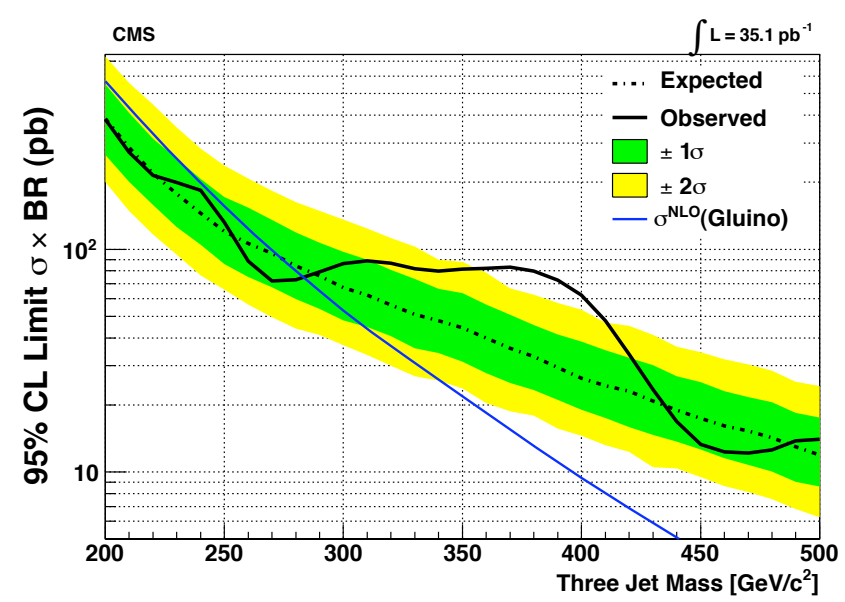

Figure 2: Expected and observed cross section limits at 95\% CL for gluino-pair production through RPV decays. Also shown are the $\pm 1 \sigma$ and $\pm 2 \sigma$ bands on the expected limit, as well as the theoretical cross section for gluino production.

CDF exclusion of $0.3<M(W)<0.8 \mathrm{TeV}$ from the dijet mass spectrum [7].

\section{Searches for Three-Jet Resonances in a Six Jet Final State}

This study is focused on a search for narrow resonances in a three jet mass spectrum using a six jet final state [8]. Variations of technicolor models, resulting in heavy colored fermions that transform as octets under $S U(3)$ color, have been proposed in a variety of forms. Other more recent models incorporate $R$-parity violating (RPV) decays of supersymmetric gluinos to three-quark final states, where the gluino represents a colored adjoint Majorana fermion. The data used in this study correspond to an integrated luminosity of $36 \pm 4 \mathrm{pb}^{-1}$.

The $p_{T}$ of the six jets is required to be greater than $45 \mathrm{GeV}$. Only two of the 20 triple-jet combinations from the six jet final state correspond to real signal, thus 18 combinations are additional background in addition to the QCD background. To effectively reduce this large background, we require each jet triplet to satisfy the following relation:

$$
M_{j j j}<\sum_{i=1}^{3}\left|p_{T}^{\mathrm{jet}}\right|_{i}-130 \mathrm{GeV}
$$

where $M_{j j j}$ is the triplet invariant mass, $\sum_{i=1}^{3}\left|p_{T}^{\text {jet }}\right|_{i}$ the scalar sum $p_{T}$ of the jets in the triplet, and $\Delta$ an offset adjusted to optimize signal sensitivity. No significant excess is observed.

The expected and observed 95\% CL upper limits as a function of mass are presented in Fig. 2. The production of gluinos decaying through the $u d s$ RPV coupling is excluded for masses between 200 and $280 \mathrm{GeV} / c^{2}$ at $95 \% \mathrm{CL}$.

\section{Search for Black Holes}

This study is focused on the search for a signature of black holes from multiple hardronic final states. In this analysis we focus on black hole production in the ADD model (semiclassical 
approximation) and in a model with string balls [9] . The data used in this study correspond to an integrated luminosity of $1.09 \pm 0.07 \mathrm{fb}^{-1}$.

The search for production of black holes is focused on a comparison of the $S_{T}$ variable to its prediction, where the $S_{T}$ variable is the scalar sum of the $E_{T}$ of individual jets, electrons, photons, MET, and muons with $E_{T}>50 \mathrm{GeV}$. The prediction of the $S_{T}$ distribution is obtained from data in the control region with $N=2$ and $N=3$ between 800 and $2500 \mathrm{GeV}$ where no evidence for new physics has been observed in the dijet analysis. No significant excess is observed in the signal region.

In addition to model-independent limit [9], we also set dedicated limits on black holes and string balls. These limits for semiclassical approximation black holes at the 95\% CL are shown in Fig. 3 (left). We exclude the production of black holes with a minimum mass varying from 4.0 to $5.1 \mathrm{TeV}$ for values of the multidimensional Planck scale up to $3.5 \mathrm{TeV}$ at $95 \% \mathrm{CL}$. The $95 \%$ CL limits on the string balls production cross section as function of string balls minimum mass are shown in Fig. 3 (right). We exclude the string balls production with a minimum mass from 4.1 to $4.5 \mathrm{TeV}$ depending on the model. These are the first direct limits on string balls set at hadron colliders.
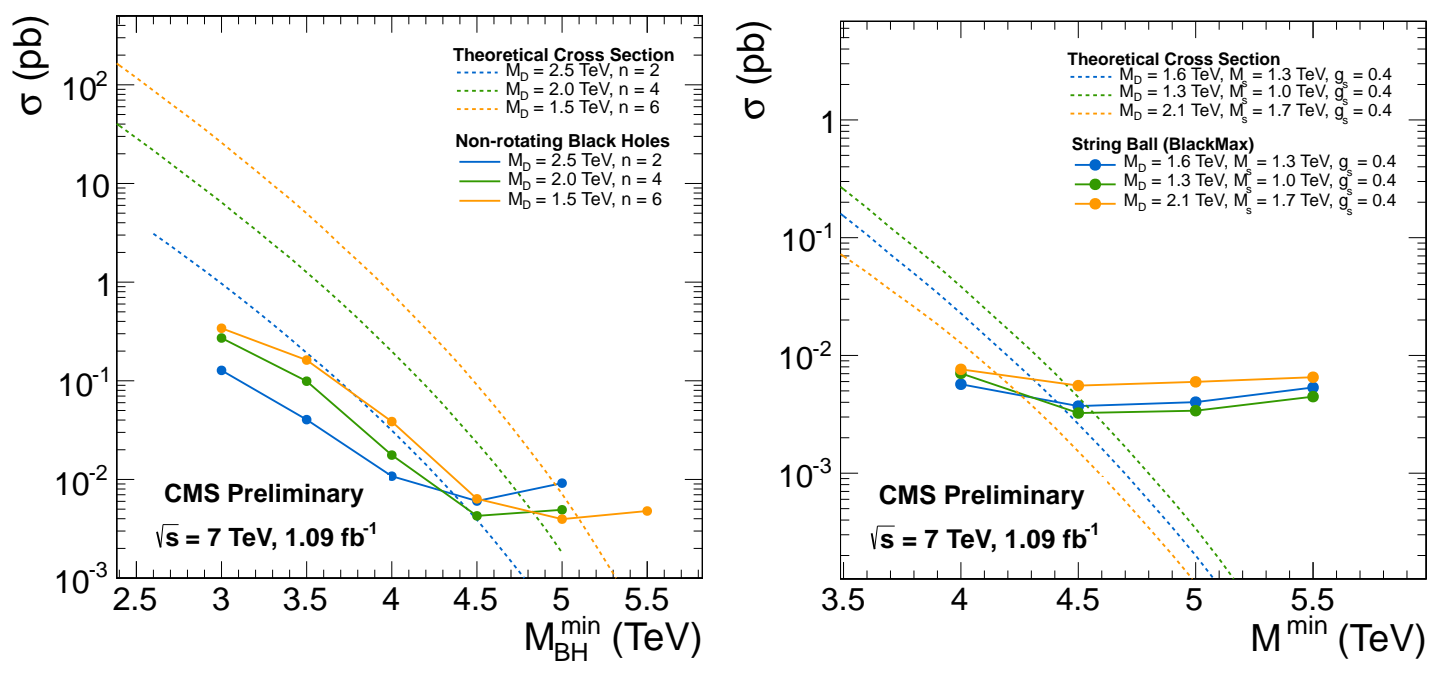

Figure 3: Cross section limits at 95\% confidence level from the counting experiments optimized for various black hole parameter sets (left) and various string ball parameter sets (right) compared with signal production cross section. Colored solid lines show experimental cross section limits, while dotted lines represent corresponding signal cross sections.

\section{Conclusion}

We performed searches for new physics in the hadronic final states. No sign for new physics is found. Upper limits are set for various models and significant parameters space are excluded.

\section{References}

[1] The CMS Collaboration, CMS PAS PFT-10-001. 
[2] The CMS Collaboration, JINST 3, S08004 (2008).

[3] Cacciari, Matteo and Salam, Gavin P. and Soyez, Gregory, Journal of High Energy Physics 2008 (2008), no. 04, 063.

[4] The CMS Collaboration, CMS PAS JME-10-010.

[5] The CMS collaboration, arXiv:1106.4775 [hep-ex], accepted by Phy. Rev. Lett.; The CMS Collaboration, CMS PAS EXO-11-059.

[6] The CMS collaboration, Phy. Rev. Lett. 105, 211801 (2010); The CMS collaboration, Phy. Lett. B 704, 123-142 (2011).

[7] The CDF Collaboration, Phys. Rev. D 79, 112002 (2009).

[8] The CMS Collaboration, Phys. Rev. Lett. 107, 101801 (2011).

[9] The CMS Collaboration, Phys. Lett. B697, 434-453 (2011); The CMS Collaboration, CMS PAS EXO-11-071. 